\title{
Bulk and Spectral Observables in Lattice QCD
}

\author{
T Hatsuda \\ Department of Physics, The University of Tokyo, Tokyo 113-0033, Japan \\ E-mail: hatsuda@phys.s.u-tokyo.ac.jp
}

\begin{abstract}
We review recent developments in lattice siumulations of the equation of state, order of the thermal phase transition and the determination of the pseudocritical temperature in $(2+1)$-flavor QCD. Owing to the increasing computer power, new argothithms, and improved fermion formulations, studies of bulk QCD matter are approaching to the stage of precision science. We also review recent lattice studies on the spectral properties of heavy quarkoniums inside the quark-gluon plasma (QGP). Although they are still in an exploratory stage, interesting physics in relation to the strongly correlated QGP is being extracted.
\end{abstract}

\section{Introduction}

One of the main goals of the lattice QCD studies is to make first principle analysis of hot/dense QCD and to supply reliable inputs to quark-gluon plasma (QGP) phenomenologies [1]. Shown in Fig:1 is a schematic QCD phase diagram indicating three basic phases; the chiral symmetry broken $(\chi \mathrm{SB})$ phase, the color superconducting (CSC) phase and the QGP phase. Precise locations of the phase boundaries and the

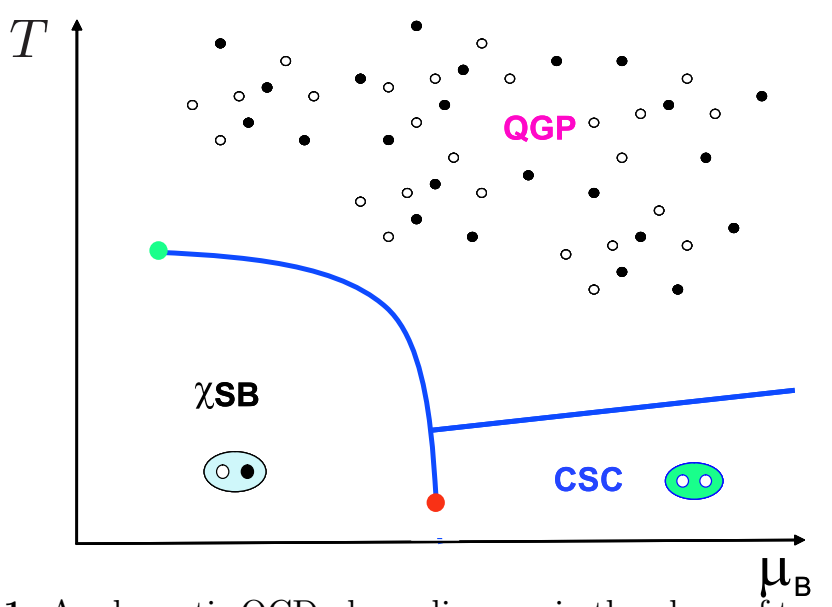

Figure 1. A schematic QCD phase diagram in the plane of temperature $T$ and baryon chemical potential $\mu_{\mathrm{B}}$. Solid lines indicate the first order phase boundaries. Several critical points at which the first order lines terminate may exit, e.g. the high $T$ critical point 2] and high $\mu_{\mathrm{B}}$ critical point [3]. 
critical points as well as the dynamics in each phase should be determined by nonperturbative method such as the lattice QCD.

QCD partition function in a finite box with a spatial volume $V$ and the lattice spacing $a$ can be written as

$$
Z(T, \mu ; a, V)=\operatorname{Tr}\left[\mathrm{e}^{-(\hat{H}-\mu \hat{N}) / T}\right]=\int[d U] F(U) \mathrm{e}^{-S_{g}(U)},
$$

where $U$ is the gauge field defined as an element of $S U(3)_{\text {c }}$ and $F(U)$ denotes the quark contribution to $Z$. Eventually, we need to take the continuum limit $(a \rightarrow 0)$ and the thermodynamic limit $(V \rightarrow \infty)$ to extract physical observables in the real world.

In the past few years, there have been considerable progress in lattice QCD approach: (i) available computer speed becomes as fast as 50 Tflops, (ii) improved fermion formulations have been tested extensively (such as staut, asqtad and p4 improved actions for staggered quarks and the clover action for Wilson quark), (iii) new fermion formulations with good chiral properties are started to be used (such as domain wall and overlap fermions), and (iv) new algorithms for full QCD simulations are proposed and implemented (such as the rational hybrid Monte Carlo method (RHMC) and the domain-decomposition hybrid Monte Carlo method (DDHMC) [5]). Shown in Fig 2 is so called the Berlin wall plot where the computational cost in unit of Tflops·year is plotted against $m_{\pi} / m_{\rho}$. The quark mass dependence of the cost becomes weaker than before, and simulations with realistic quark masses may be done in a reasonable amount of time by using $\mathrm{O}(50)$ Tflops machines.

In the following, we will focus on the bulk and spectral properties associated with the QCD phase transition at finite $T$ with $\mu_{\mathrm{B}}=0$. For the developments in lattice QCD at finite chemical potential, see the recent review [4].

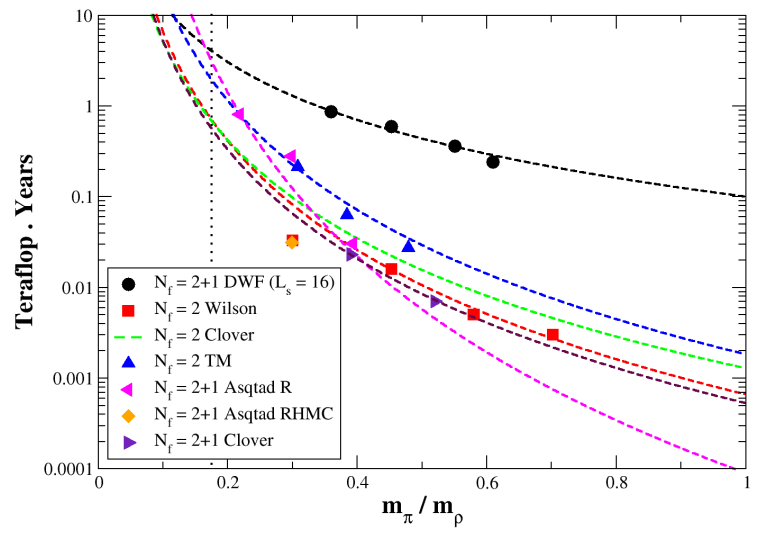

Figure 2. Updated Berlin wall plot which shows the cost to generate 1000 independent gauge configurations on $24^{3} \times 40$ lattice with $a=0.08 \mathrm{fm}$ in various fermion formulations with modern algorithms $[6]$. 


\section{Equation of state in $(2+1)$-flavor QCD}

The equation of state at finite $T$ characterizes the bulk QCD matter. Also it becomes an fundamental input together with the transport coefficients [7] to the relativistic hydrodynamics for QCD fluid [8]. Shown in Fig 3 is the state-fo-the-art calculation of the energy density $\varepsilon$ as a function of $T$ in $(2+1)$-flavor QCD with asktad improved staggered quarks. (See also the recent results for 3-flavor QCD with the p4 action 10] and for $(2+1)$-flavor QCD with the stout action [11].)

The figure shows that $\varepsilon / T^{4}$ increases rapidly at $T \sim 200 \mathrm{MeV}$ and approaches the Stefan-Boltzmann (SB) limit from below. The deviation from the SB limit at high $T$ is due to the quark-gluon interaction which decreases only logarithmically as $T$ increases. Quantitative understanding of the deviation is a non-trivial issue and cannot be treated in naive thermal perturbation [13].

\section{Order of the transition in $(2+1)$-flavor QCD}

Using the pressure $P(\vec{K})=(T / V) \cdot \ln Z$ and a vector $\vec{K} \equiv\left(T, \mu, m_{q}, a, V, \cdots\right)$, one may define the $n$-th order transition as the one where non-analyticity of $P$ in the thermodynamic limit $(V \rightarrow \infty)$ first appears in the $n$-th derivative of $P$ with respect to $\vec{K}$. For example, if $(\partial / \partial T) P=s$ is discontinuous at certain $T$, it is the first order transition. On the other hand, if $(\partial / \partial T) P$ is continuous and $(\partial / \partial T)^{2} P=(\partial / \partial T) s=$ $c_{V} / T$ diverges at certain $T$, it is an example of the second order transition. If smooth

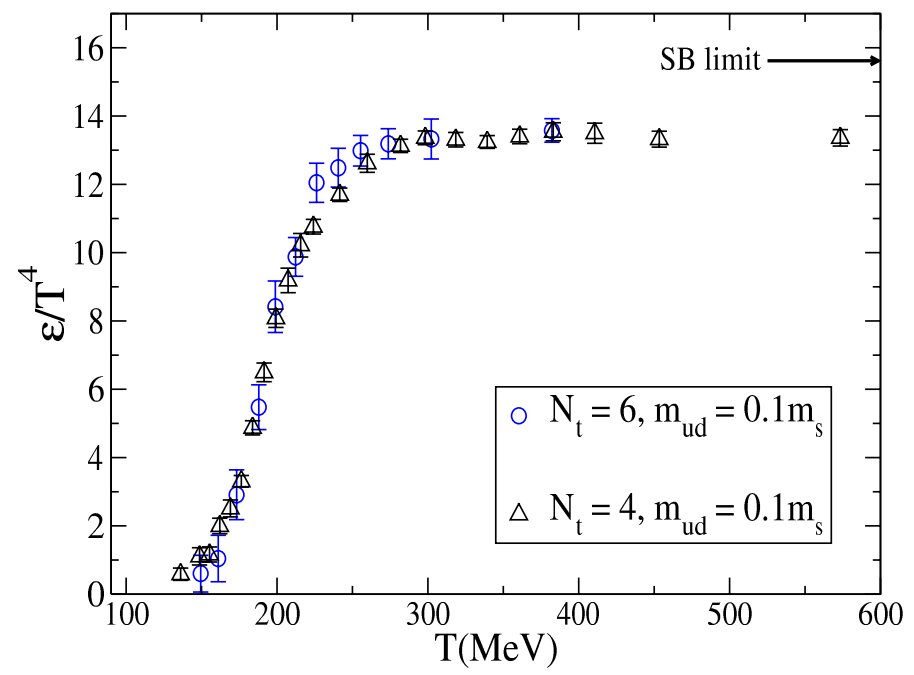

Figure 3. Energy density $\varepsilon$ as a function of $T$ for $(2+1)$-flavor QCD with asqtad improved action on the lattice, $N_{t} \times N_{s}^{3}=4 \times\left(12^{3}-16^{3}\right)$ and $6 \times\left(12^{3}-20^{3}\right)[9$. The strange quark mass $m_{\mathrm{s}}$ is about the physical value, while the light quark mass $m_{\mathrm{ud}} \simeq 0.1 m_{s}$ corresponding to $m_{\pi} / m_{\rho} \simeq 0.3$. The Sommer scale $r_{1}=0.138(7)(4)$ $\mathrm{fm}$ is used to set the scale. Lattice spacing at $T \simeq 200 \mathrm{MeV}$ is $0.247(0.165) \mathrm{fm}$ for $N_{t}=4$ (6). 
phase change takes place without the non-analyticity of $P$, it is the crossover. To make a distinction between the phase transition and the crossover from the numerical simulations, the scaling behavior of certain observables as a function of $V$ needs to be studied (the finite size scaling analysis) [17]. In the (2+1)-flavor QCD, the relevant observable is the chiral susceptibility whose volume dependence at its maximum reads

$$
\chi_{m}=\left(\partial / \partial m_{\mathrm{ud}}\right)^{2} P \rightarrow V^{\alpha} \text { at the peak, }
$$

where $\alpha \simeq 1(2 / 3)$ for the first (second) order transition, and $\alpha \simeq 0$ for crossover.

Shown in the left panel of Fig 4 is the dimensionless chiral susceptibility $\chi_{m} / T^{2}$ around its peak for different values of the dimensionless volume $N_{s}=V / a^{3}=$ $18^{3}, 24^{3}, 32^{3}$ for $(2+1)$-flavor QCD with realistic masses of $\mathrm{u}, \mathrm{d}, \mathrm{s}$ quarks [12]. There is no appreciable volume dependence for the height of the susceptibility, which suggests the crossover nature. The right panel of Fig, 4 shows the subtracted chiral susceptibility in the continuum limit $\Delta \chi\left(=\right.$ the peak of $\left.\chi_{m}(T)-\chi_{m}(0)\right)$ in a renormalization group invariant combination $T^{4} /\left(m_{\mathrm{ud}}^{2} \Delta \chi\right)$ as a function of the dimensionless inverse-volume, $1 /\left(T_{\mathrm{c}}^{3} V\right)$. The height of the susceptibility approaches to a non-vanishing constant in the thermodynamic limit and has different scaling behavior from those of first or second order transitions. This gives a strong evidence that the hadron-QGP transition at finite $T$ with zero chemical potential is a crossover in the real world.
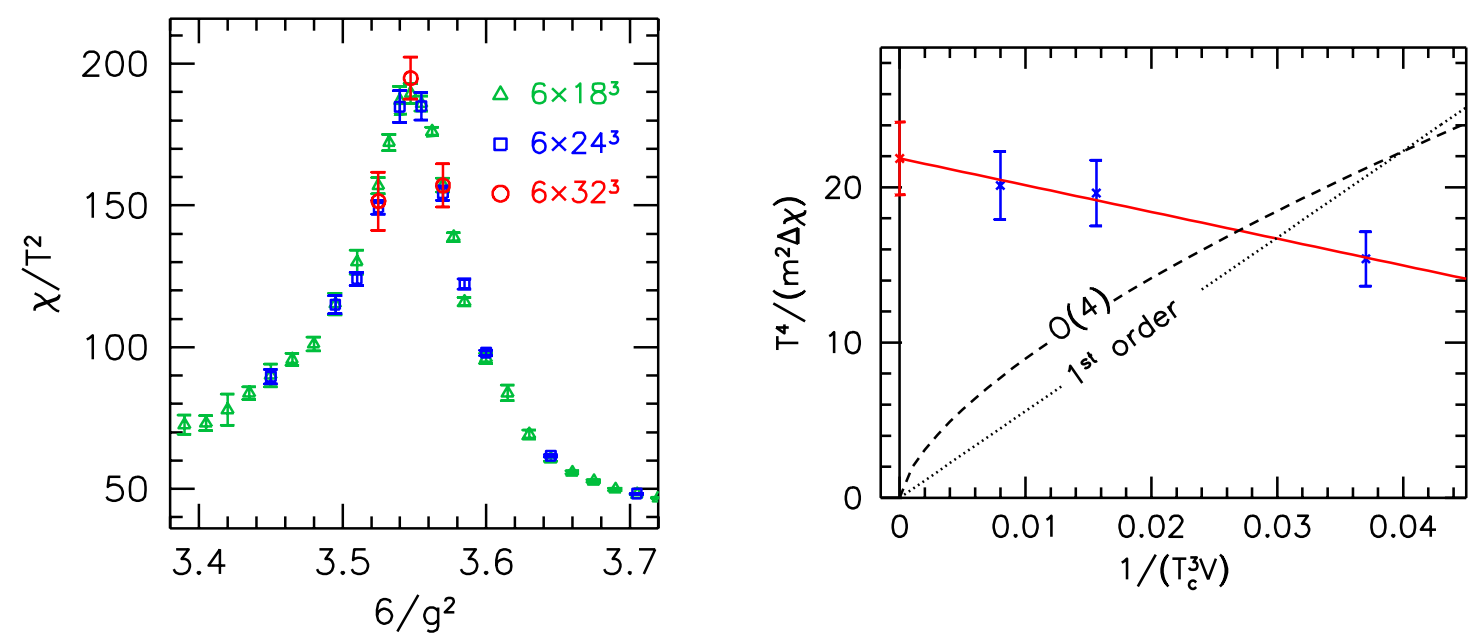

Figure 4. Left: The chiral susceptibility in $(2+1)$-flavor QCD as a function of the bare coupling, $6 / g^{2}$, for different values of the spatial volume, $N_{t}^{3}=V / a^{3}=18^{3}, 24^{3}, 32^{3}$. The stout improved staggered fermion action is employed and the quark masses are chosen to reproduce $m_{K} / m_{\pi}=3.689$ and $f_{K} / m_{\pi}=1.185$. Right: The volume dependence of the peak of the subtracted chiral susceptibility in the continuum limit. Dotted (dashed) line is an expectation from the first order (second order) phase transition. Figures are taken from [12]. 


\section{Pseudo-critical temperature for (2+1)-flavor QCD}

Since the hadron-QGP transition in the real world is crossover, the "critical" temperature $T_{\mathrm{c}}$ is not a well-defined concept. Nevertheless, one may introduce a "pseudo-critical" temperature $T_{\mathrm{pc}}$ as a peak position of certain susceptibility. The actual value of $T_{\mathrm{pc}}$ is different for different choice of susceptibility, e.g. $\chi_{m}=\left(\partial / \partial m_{\mathrm{ud}}\right)^{2} P$, $\chi_{T}=(\partial / \partial T)^{2} P$, and $\chi_{m T}=\left(\partial^{2} / \partial m \partial T\right) P$. Note also that multiplying an arbitrary function of $m_{\mathrm{ud}}$ and $T$ to the susceptibilities can change the value of $T_{\mathrm{pc}}$.

Shown in Fig 5 is a summary of (pseudo-)critical temperature recently calculated from the dimensionless chiral susceptibility, $\chi_{m} / T^{2}$, for $(2+1)$-flavor QCD. Extrapolation to the continuum limit is taken by using the data for several values of $N_{t}$. The upper two bars are $T_{\mathrm{c}}$ with different extrapolations to the chiral limit $m_{\mathrm{ud}}=0$ by MILC collaboration [14]. The middle bar is $T_{\mathrm{pc}}$ extrapolated to the realistic $m_{\mathrm{ud}}$ by RBC-Bielefeld collaboration [15]. The lower bar is $T_{\mathrm{pc}}$ calculated at the realistic $m_{\text {ud }}$ by Wuppertal-Budapest collaboration [16]. Note that different improved actions and different scale determinations are adopted in three groups. When extracting $T_{\mathrm{pc}}$ in $\mathrm{MeV}$ unit, we need to use the chain rule:

$$
T_{\mathrm{pc}}=\left(\frac{T_{\mathrm{pc}}(a)}{a^{-1}}\right)_{a \rightarrow 0} \cdot\left(\frac{a^{-1}}{M(a)}\right)_{a \rightarrow 0} \cdot M \text { "exp" } .
$$

Here $M$, which has a dimension of mass, is chosen either to be a direct physical observable (such as $m_{\rho}, f_{\pi}$ and $f_{K}$ ) or to be a semi-empirical quantity (such as the Sommer scales $1 / r_{0}$ and $1 / r_{1}$ defined from the heavy-quark potential through $r^{2} d V(r) /\left.d r\right|_{r=r_{0}}=1.65$ and $\left.r^{2} d V(r) /\left.d r\right|_{r=r_{1}}=1.0\right)$.

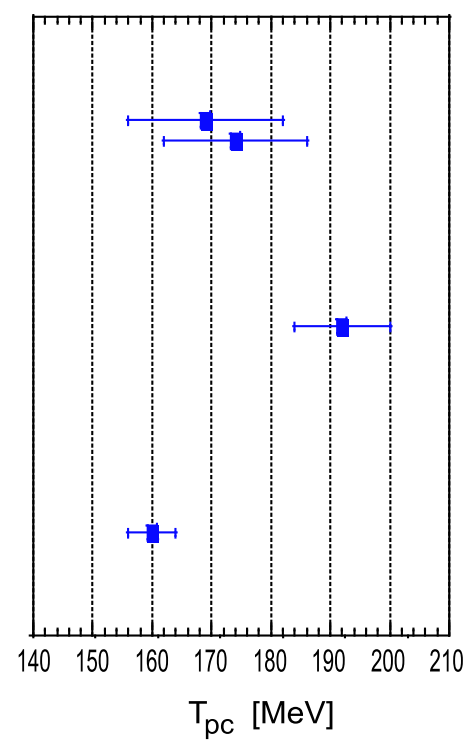

MILC Coll., Phys.Rev.D71 ('05) 034504

169 (12)(4) MeV at $\mathrm{m}_{\mathrm{ud}}=0$

174 (11)(4) MeV at $\mathrm{m}_{\mathrm{ud}}=0$

$\mathrm{N}_{\mathrm{t}}=4,6,8, \mathrm{~N}_{\mathrm{s}} / \mathrm{N}_{\mathrm{t}}=2$

action: asqtad, scale: $r_{1}=0.317(7) \mathrm{fm}$

RBC-Bielefeld Coll., Phys.Rev.D74 ('06) 054507

192(7)(4) MeV at physical point

$\mathrm{N}_{\mathrm{t}}=4,6, \mathrm{~N}_{\mathrm{s}} / \mathrm{N}_{\mathrm{t}}=2-4$

action: $\mathrm{p} 4 \mathrm{fat} 3$, scale $\mathrm{r}_{0}=0.469(7) \mathrm{fm}$

Wuppertal-Budapest Coll., Phys.Lett.B643 ('06) 46

$151(3)(3)+9 \mathrm{MeV}$ at physical point

$\mathrm{N}_{\mathrm{t}}=, 6,8,10, \mathrm{~N}_{\mathrm{s}} / \mathrm{N}_{\mathrm{t}}=4$

action: stout, scale: $\mathrm{f}_{\mathrm{K}}=159.8 \mathrm{MeV}$

Figure 5. Recent determinations of the (pseudo-)critical temperature for (2+1)flavor QCD with staggered quarks. As for the lowerst bar, we add $9 \mathrm{MeV}$ to make a comparison to other results obtained from $\chi_{m} / T^{2}[16$. 
There are a number of issues to be studied further before drawing definite conclusion on the value of $T_{\mathrm{pc}}$ in the real world. In particular, (i) careful continuum extrapolation $\left(a \rightarrow 0\right.$ ) by the data with larger $N_{t}$, (ii) determination of $T_{\mathrm{pc}}$ in the case of Wilson fermion [18], and (iii) resolving the difference between the Sommer scale in the continuum limit obtained from $a$ being determined by the 2S-1S mass splitting of the bottomonium with staggered quark $\left(r_{0}=0.468(7) \mathrm{fm}\right)$ [19] and that obtained from $a$ being determined by the kaon mass with Wilson quark $\left(r_{0}=0.516(21) \mathrm{fm}\right)$ [20].

\section{Heavy quarks inside QGP}

Heavy quarks in the real world (such as the charm and bottom) have finite masses and may receive substantial kick from the light plasma-constituents which have typical energy of about 3T. Therefore, it is important to examine the dynamical correlations (both spatial and temporal) of the heavy quarks inside QGP. It is also relevant to study the modification of the heavy quarkonium properties in hot QCD matter [21].

\subsection{Charmonium wave function in quenched $Q C D$}

An attempt to study the $J / \Psi$ wave function at finite temperature in quenched QCD $(F(U)$ is take to be unity in Eq.(1) ) on an anisotropic lattice was performed by Umeda et al. [22]. They have studied the equal-time Bethe-Salpeter wave function $w(\vec{r}, \tau)$ at finite $T$ in the Coulomb gauge. In Fig, 6 , the normalized wave function, $\phi(\vec{r}, \tau)=w_{i}(\vec{r}, \tau) / w_{i}(\overrightarrow{0}, \tau)$ is plotted below and above $T_{c}$ for various time slices. The black dashed line is the wave function prepared at $\tau=0$. For free quarks without gauge

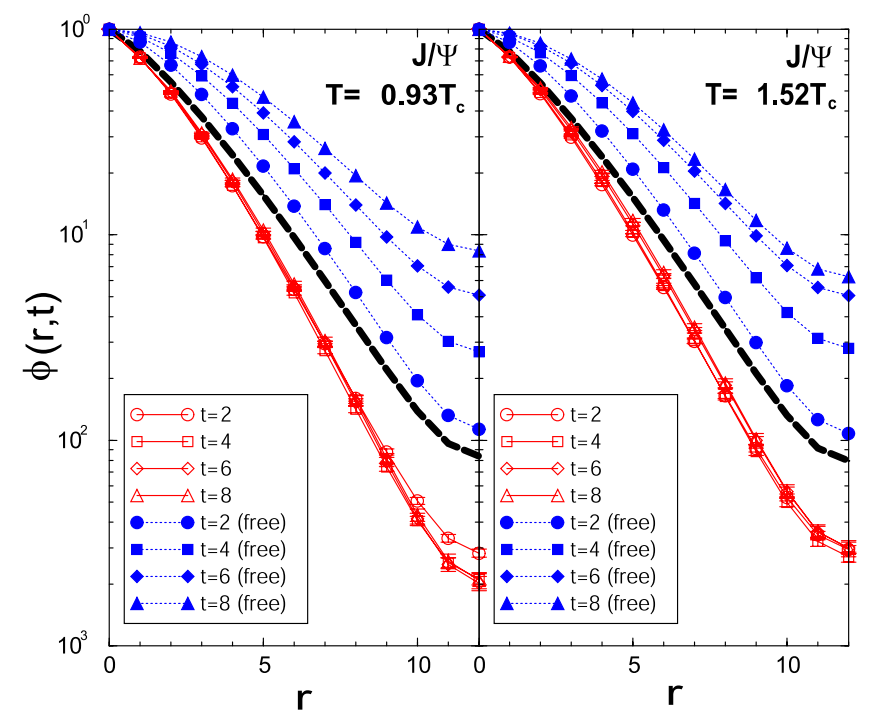

Figure 6. The equal-time Bethe-Salpeter wave function normalized at the origin as a function of the spatial separation $r$ between the charm and the anti-charm. Blue (red) points correspond to free (interacting) quarks [22]. The black dashed line is the wave function initially prepared at $\tau=0$. Note that $t \equiv \tau$ in this figure. 
interactions, the wave function becomes broader as the imaginary time $\tau$ increases. On the other hand, the wave function for the interacting system is almost unchanged even at $T=1.52 T_{c}$, which suggests that $J / \Psi$ may survive as a bound state in the deconfined phase.

\subsection{Charmonium spectral function in quenched $Q C D$}

The spectral functions of hadronic correlators give us another information on hadronic modes at finite $T$ as originally suggested in [23]. The spectral function $\sigma(\omega, \vec{p})$ for hadronic correlation may be defined through the spectral decomposition:

$$
D(\tau, \vec{p})=\int_{-\infty}^{+\infty} \frac{\mathrm{e}^{-\tau \omega}}{1 \mp \mathrm{e}^{-\omega / T}} \sigma(\omega, \vec{p}) d \omega \quad\left(0 \leq \tau<T^{-1}\right) .
$$

The maximum entropy method (MEM) provides an efficient and powerful way to obtain a unique $\sigma$ from the lattice data $D$ without making a priori parameterization [24].

Applications of MEM to the $\bar{s}$ mesons at finite $T$ [25] and to the charmoniums at finite $T$ [26, 27, 28, 29] within the quenched QCD have been carried out and it was shown that the $J / \Psi$ and $\eta_{c}$ survive even above $T_{c}$ as distinct peaks. Shown in the left (right) panel of Fig.7 is the dimensionless spectral function for $J / \Psi\left(\eta_{\mathrm{c}}\right)$ below and above $T_{c}$ on $32^{3} \times(96-32)\left(24^{3} \times(160-34)\right)$ anisotropic lattices with the spatial volume $V=(1.25 \mathrm{fm})^{3}\left(V=(1.34 \mathrm{fm})^{3}\right)$ taken from Ref.[26] (Ref.[29]). The figures indicate that the low-lying s-wave resonances still survive even at $1.5 T_{c}$, which is consistent with the result in Fig 6. Note that this result is not an artifact of the small spatial volume, which was carefully checked in [30].

There are several topics which are interesting to be studied within the quenched QCD, such as the spectral functions of the charmoniums moving inside the hot medium [31] and the spectral functions of bottomoniums above $T_{\mathrm{c}}$ [32].
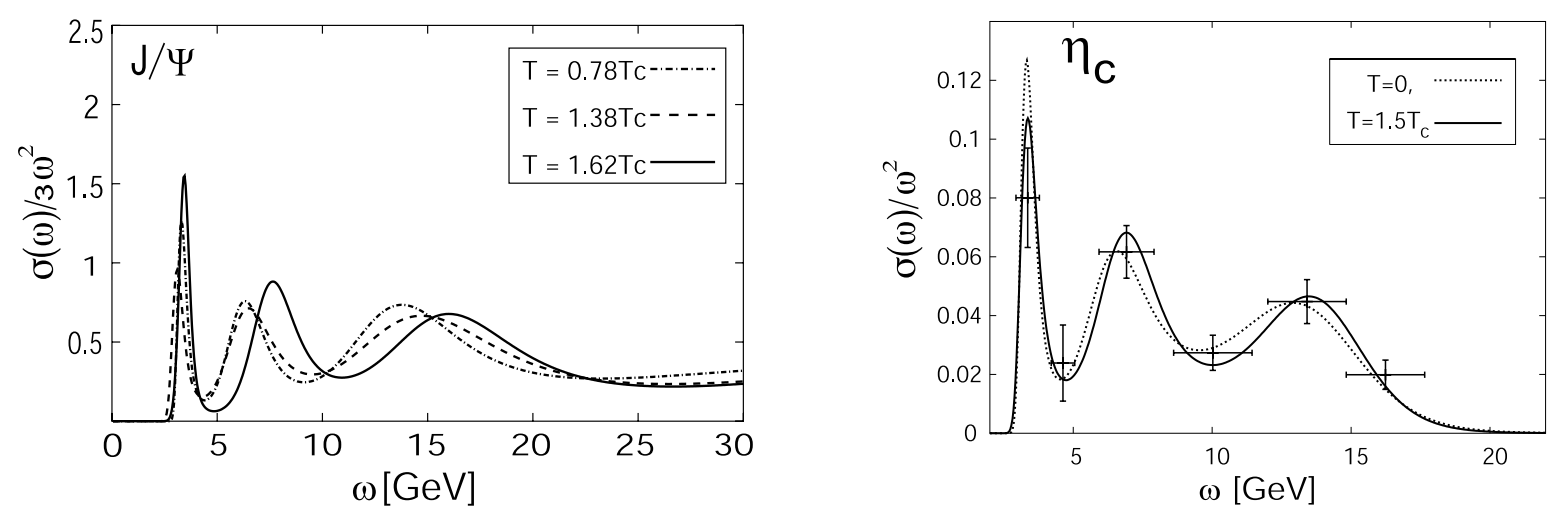

Figure 7. Left: Spectral functions measured in quenched QCD simulations on an anisotropic lattice with the spatial volume $(1.25 \mathrm{fm})^{3}$ for the $J / \Psi$ channel [26]. Right: Spectral functions measured in quenched QCD simulations on an anisotropic lattice with the spatial volume $(1.34 \mathrm{fm})^{3}$ for the $\eta_{c}$ channel [29]. 

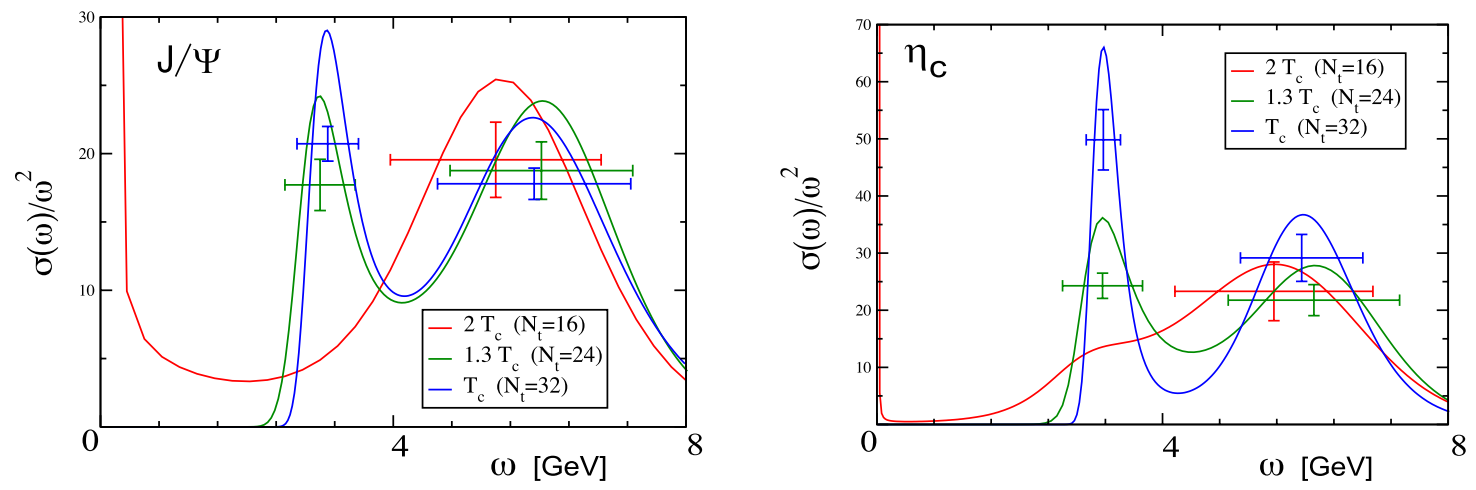

Figure 8. Spectral functions in the vector and pseudo-scalar channels in 2-flavor QCD on $8^{3} \times(32-16)$ anisotropic lattices. The spatial lattice volume is $V \sim(1.2 \mathrm{fm})^{3}$ and the light quark mass corresponds to $m_{\pi} / m_{\rho} \sim 0.5$. The figures are adapted from 34 .

\subsection{Charmonium spectral function in 2-flavor $Q C D$}

In quenched QCD, only gluons are thermally excited in the plasma, while, in full QCD, both quarks and gluons are thermally active. Because the number of degrees of freedom increase in full QCD, the dissociation rate of the chamoniums will be larger. On the other hand, the pseudo-critical temperature $T_{\mathrm{pc}}$ in full QCD (160-200 MeV) is substantially smaller than $T_{\mathrm{c}} \sim 270 \mathrm{MeV}$ in quenched QCD. Due to the compensation of these effects, the ratio of the dissociation temperature $T^{*}$ and the (pseudo-)critical temperature in full QCD is not much different from the ratio in quenched QCD and could take a value of around 2 [33].

Aarts et al. has recently reported an exploratory study of 2-flavor QCD on $8^{3} \times(32-16)$ anisotropic lattices [34]. Shown in Fig 8 are the spectral functions of the $J / \Psi$ and $\eta_{\mathrm{c}}$. The results suggest that, even with the thermal excitations of light quarks, the low-lying charmoniums may survive inside the quark-gluon plasma above $T_{\mathrm{c}}$ until they dissociate away around $2 T_{\mathrm{pc}}$.

\section{Summary}

We reviewed recent developments in lattice simulations on the equation of state, crossover nature of the thermal phase transition and the determination of the pseudocritical temperature in (2+1)-flavor QCD. Recent lattice results on the spectral properties of the heavy quarkoniums inside the quark-gluon plasma are also summarized.

Acknowledgements: This work was partially supported by Japanese MEXT Grant No. 18540253. The author thanks T. Umeda, S. Ejiri, F. Karsch and Z. Fodor for useful information and discussions. 


\section{References}

[1] Yagi K, Hatsuda T and Miake Y 2005 Quark-Gluon Plasma, (Cambridge Univ. Press, Cambridge).

[2] Asakawa M and Yazaki K 1989 Nucl. Phys. A504 668. Barducci A et al. 1989 Phys. Lett. B231 463.

[3] Hatsuda T, Tachibana M, Yamamoto N, and Baym G 2006 Phys. Rev. Lett. 97122001.

[4] Schmidt C 2006 PoS LAT2006 021 hep-lat/0610116.

[5] Kennedy A D 2006 arXiv: hep-lat/0607038. Del Debbio L et al. 2006 arXiv: hep-lat/0610059.

[6] Clark M A 2006 arXiv: hep-lat/0610048.

[7] Sakai S and Nakamura A 2006 PoS LAT2005 186 hep-lat/0510100.

[8] Hirano T and Gyulassy M 2006 Nucl. Phys. A769 71.

[9] Bernard C et al. 2006 arXiv: hep-lat/0611031

[10] Karsch F 2006 J. Phys. Conf. Ser. 46122.

[11] Aoki Y, Fodor Z, Katz S D and Szabo K K 2006 JHEP 0601089.

[12] Aoki Y, Endrodi G, Fodor Z, Katz S D, and Szabo K K 2006 Nature 443675.

[13] Kraemmer U and Rebhan A 2004 Rept. Prog. Phys. 67351.

[14] Bernard C et al. 2005 Phys. Rev. D71 034504.

[15] Cheng M et al. 2006 Phys. Rev. D74 054507.

[16] Aoki Y, Fodor Z, Katz S D, and Szabo K K 2006 Phys. Lett. B643 46.

[17] Ukawa A 1993 Lectures on Lattice QCD at Finite Temperature, (Uehling Summer School, Seattle).

[18] Maezawa Y et al. 2007 arXiv: hep-lat/0702005.

[19] Gray A et al. (HPQCD and UKQCD Coll.) 2005 Phys. Rev. D72 094507.

[20] Ishikawa T et al. (CP-PACS and JLQCD Coll.) 2006 PoS LAT2006 181 hep-lat/0610050.

[21] Matsui T and Satz H 1986 Phys. Lett. B178 416.

Hashimoto T, Hirose K, Kanki T and Miyamura O 1986 Phys. Rev. Lett. 572123.

[22] Umeda T, Katayama R, Miyamura O and Matsufuru H 2001 Int. J. Mod. Phys. A16 2215.

[23] Hatsuda T and Kunihiro T 1985 Phys. Rev. Lett. 55 158. DeTar C 1985 Phys. Rev. D32 276.

[24] Asakawa M, Hatsuda T and Nakahara Y 2001 Prog. Part. Nucl. Phys. 46459.

[25] Asakawa M, Hatsuda T and Nakahara Y 2003 Nucl. Phys. Proc. Suppl. 119481.

[26] Asakawa M and Hatsuda T 2004 Phys. Rev. Lett. 92 012001; J. Phys. G30 S1337.

[27] Datta S, Karsch F, Petreczky P and Wetzorke I 2004 Phys. Rev. D69 094507.

[28] Umeda T, Nomura K and Matsufuru H 2005 Eur. Phys. J. C39S1 9.

[29] Jakovac A, Petreczky P, Petrov K and Velytsky A 2007 Phys. Rev. D75 014506.

[30] Iida H, Doi T, Ishii N, Suganuma H and Tsumura K 2006 Phys. Rev. D74 074502.

[31] Datta S, Karsch F, Wissel S, Petreczky P and Wetzorke I 2004 arXiv: hep-lat/0409147. Aarts G, Allton C, Foley J, Hands S and Kim S 2006 arXiv: hep-lat/0610061.

[32] Datta S, Jakovac A, Karsch F and Petreczky P 2006 AIP Conf.Proc. 84235 hep-lat/0603002.

[33] Hatsuda T T 2006 Int. J. Mod. Phys. A21 688 hep-ph/0509306.

[34] Aarts G et al. 2006 PoS LAT2006 126 [hep-lat/0610065. 